\title{
Color Correction in Portable-type Urine Analyzer
}

\author{
Jae-Hyung Kim and Chang Hee Park \\ Department of Physics, Inje University, Kimhae, Kyungnam 621-749, Korea \\ Seung Jin Lee \\ Institute of Medical Development, SEAN Imagination Technology Co.,Ltd., Pusan 601-827, \\ Korea \\ Gye Rok Jeon* and Gi Ryon Kim \\ Department of Biomedical Engineering, College of Medicine, Pusan National University, \\ Pusan 602-739, Korea \\ E-Mail: grjeon@pusan.ac.kr
}

(Received 2 October 2002, Accepted 9 December 2002)

\begin{abstract}
Color correction methods of chromaticity coordinates using Color Matching Function (CMF) were studied to develop a device-independent portable-type urine analyzer. The reflection spectra were measured for the degrees of 10 test items of the urine reagent strip (urine strip) to develop a portable-type urine analyzer. A computer simulation was performed to quantitatively distinguish the color reactions of the urine system, by using the spectral power distribution of Light Emitting Diode(LED), the reflection of a urine strip, and spectral sensitivity of a photodiode. To develop a device-independent system, chromaticity coordinates were modified to reduce the color deviations in the urine strip, by using the temperature compensation of LED and the color transformation by CMF. The experimental values obtained by developed urine system exhibited the accuracy above $95 \%$ for all color samples.
\end{abstract}

Keywords : Urine strip, Urine analyzer, Chromaticity coordinate, Color correction

\section{INTRODUCTION}

As terminal products of protein or nucleic acid, urine contains metabolic ma terials like urea, uric acid, creatine and amino acid, and several heterogeneous materials such as hormones, vitamins and enzymes. Therefore, we can obtain the vital clinical information about the function of several organs such as the heart, the liver and the endocrine glands, as well as information about the diseases of kidney and urethra. Urine has the valuable utilities as a diagnosis and test materials of diseases since it can be easily obtained from the body without any pain to patients. In recent years, test methods combine test paper which uses the urine strip containing reagents responding to constituent compounds in urine, with photometry which detects the concentration variation of diffused light in a urine strip during the illumination of visible light to a urine strip[1].

Outputs of urine analyzer based on colorimetric model often exhibit the color distortion due to the nonlinear characteristics of their components. Color correction techniques minimizing the color distortion and problem from device dependent color spaces play a key role in obtaining accurate results. Previously, urine analyzer using fuzzy logic-based classifier and quality control method was implemented by our group[2]. Nonlinear characteristics of actual hardware were also calibrated by a neural network determining a mapping which maps the device dependent control values to a device independent color space[3].

In this study, the reflection spectra were measured for each degree of 10 test items in the urine strip to develop a portable-type urine analyzer. The spectral responses composed of the spectral power distribution of chip-LED and the spectral sensitivity of photodiode differ significantly from that composed of the standard luminant, $\mathrm{p}_{\mathrm{c}}$ and $\mathrm{CMF}$ in human vision. Therefore, computer simulations were carried out to discriminate the color reactions of the urine strip in the portable-type urine analyzer. The chromaticity coordinates obtained by using our urine system exhibited an excellent accuracy and reproducibility for all color samples. 


\section{EXPERIMENTAL}

\subsection{Measurement of reflection spectra}

Reflection spectra were measured for each degree of 10 test items in the urine strip. After the pads of urine strip became wet with the standard reagents, each degree of 10 test items is shown in Table 1. Color changes due to chemical reaction are detected by spectrophotometer (Varian Cary-5E, USA). Reflection spectra were measured at room temperature in the wavelength of 400$800 \mathrm{~nm}$

\subsection{Spectral power distribution of Chip-LED}

Chip-LED E1S10-1W (Toyoda Gosei, Japan) was used as a light source in a portable-type urine analyzer. After connecting a $220 \Omega$ resistor to the chip-LED, dc voltage was gradually increased until the saturation of light output. Light radiating from the chip-LED was focused to monochromator (Spex 1403, USA) by using a convex lens with focal length of $70 \mathrm{~mm}$. By controling diffraction grating, the spectral power distributions of incident light were measured in the wavelength of 400 $800 \mathrm{~nm}$. To investigate the temperature-dependent luminous intensity, the spectral power distributions of chip-LED were also measured in the temperature range of $11.8-24.6{ }^{\circ} \mathrm{C}$ while applying $2.0 \mathrm{~V}$ for red LED, and $3.6 \mathrm{~V}$ for green LED and blue LED.

\subsection{Spectral sensitivity of photodiode}

A white light beam from a halogen lamp with a maximum output of $80 \mathrm{~W}$ was modulated to square wave by a beam chopper (200 RPM), and then the square wave was focused on the inlet slit of the monochromator

Table 1. Test items and each degree of a urine strip.

\begin{tabular}{|c|c|c|c|c|c|c|c|}
\hline $\begin{array}{l}\text { Degree } \\
\text { Tests \& } \\
\text { Reaction time }\end{array}$ & $\begin{array}{c}1 \\
\text { neg. }\end{array}$ & 2 & 3 & 4 & 5 & 6 & 7 \\
\hline \multirow{2}{*}{$\begin{array}{l}\text { Urobilinogen } \\
30 \mathrm{sec} .(\mathrm{mg} / \mathrm{d} \mathrm{l})\end{array}$} & 0.1 & 1 & \multirow{2}{*}{2} & \multirow{2}{*}{4} & \multirow{2}{*}{8} & \multirow{2}{*}{12} & \\
\hline & \multicolumn{2}{|c|}{ normal } & & & & & \\
\hline $\begin{array}{c}\text { Glucose } \\
30 \sim 60 \mathrm{sec} \text {. }(\mathrm{mg} / \mathrm{dl})\end{array}$ & Neg. & \pm 100 & \pm 250 & $\begin{array}{c}++ \\
500 \\
\end{array}$ & $\begin{array}{r}++ \\
1000 \\
\end{array}$ & & \\
\hline $\begin{array}{c}\text { Ketones } \\
30 \sim 60 \text { sec. }\end{array}$ & Neg. & \pm & + & ++ & +++ & & \\
\hline $\begin{array}{l}\text { Bilirubin } \\
30 \sim 60 \mathrm{sec} .\end{array}$ & \multicolumn{2}{|c|}{ normal } & + & ++ & +++ & & \\
\hline $\begin{array}{c}\text { Protein } \\
\text { 30sec. }(\mathrm{mg} / \mathrm{dl})\end{array}$ & Neg. & Trace & $\stackrel{+}{30}$ & $\begin{array}{l}++ \\
100\end{array}$ & $\begin{array}{l}+++ \\
300\end{array}$ & $\begin{array}{l}++++ \\
1000\end{array}$ & \\
\hline $\begin{array}{l}\text { Nitrite } \\
30 \mathrm{sec} .\end{array}$ & Neg. & & Posit & \multicolumn{4}{|c|}{ Any pink color is positive } \\
\hline $\begin{array}{c}\mathrm{PH} \\
\text { Immediate }\end{array}$ & 5 & 6 & 6.5 & 7 & 8 & 9 & \\
\hline \multirow{2}{*}{$\begin{array}{c}\text { Blood } \\
30 \sim 60 \text { ble }\end{array}$} & \multirow[b]{2}{*}{ Neg. } & \multirow[b]{2}{*}{+} & ++ & \multirow[b]{2}{*}{+++} & + & ++ & \\
\hline & & & $\begin{array}{c}\text { Hemo } \\
\text {-lysis }\end{array}$ & & \multicolumn{2}{|c|}{ No hemolisis } & \\
\hline $\begin{array}{c}\text { Specific gravity } \\
10-30 \text { sec. }\end{array}$ & 1.000 & 1.005 & 1.010 & 1.015 & 1.020 & 1.025 & 1.030 \\
\hline $\begin{array}{l}\text { Leukocytes } \\
60 \sim 90 \mathrm{sec} .\end{array}$ & Nor-mal & trace & + & ++ & +++ & & \\
\hline
\end{tabular}

by using a convex lens with focal length of $70 \mathrm{~mm}[4,5]$. Spectrally resolved light was focused on a photodiode MBC-3026C (Moririca Electronic, Japan) after passing the monochromator. Light was converted to an electrical signal and then the electric signal was displayed by oscilloscope. Spectral sensitivity of photodiode was recorded in the wavelength range of $300-900 \mathrm{~nm}$ by a controlling PC. To investigate the effects of spectral sensitivity due to temperature variation, the spectral sensitivity of photodiode was measured with increasing temperature up to $25^{\circ} \mathrm{C}$.

\subsection{Fabrication of optic module}

To fabricate the portable-type urine analyzer, a small optic module is composed of chip-LED and photodiode. Chip-LED E1S10-1W was used as a light source in the light detecting part of the urine system. Chip-LED E1S10-1W has a small size ( $3 \mathrm{~mm} \times 3 \mathrm{~mm}$ ) with $\mathrm{R}, \mathrm{G}, \mathrm{B}$ pads, as well as high luminous intensity. The minimum luminous intensity of chip-LED are $60-300 \mathrm{mcd}$. Si photodiode MBC-3026C was used as a light detecting sensor. It is considered that the visible spectrum filter built in MBC-3026C can remarkably reduce the temperature effects from external surroundings. Photodiode MBC-3026C exhibited a low dark current of $0.01-10 \mathrm{pA}$ and wide luminous range of $0.01-10,000$ lx.

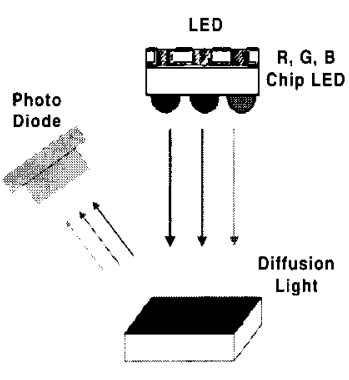

(a)

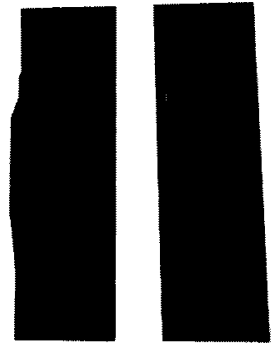

(b)
Fig. 1. (a) Structure of the optic module composed of the chip LED and the photodiode, (b) photograph of a fabricated optic module.

\section{RESULTS AND DISCUSSION}

\subsection{Reflection spectra of urine strip}

Reflection spectra were measured for the each degree of 10 test items in a urine strip. This data for all test items was also published in the literature by our group[6]. As a example, Figure 2 shows the reflection spectra of glucose which were measured at $35 \mathrm{~s}$ and $45 \mathrm{~s}$ after a urine pad of glucose became wet with standard reagent with 5 degrees. Glucose is the test item for examining 
glucose, pancreatitis, cerebral haemorrhage, hyperthyroidism, and pregnancy. The degrees of glucose were divided into neg., $\pm 100, \pm 250, \pm 500$, and \pm 1000 . The reflection of glucose was decreased in the wavelength region of $400-600 \mathrm{~nm}$ with respect to the increase of test degree. Whereas the reflection of negative degree was high in the wavelength of $450-550$ $\mathrm{nm}$, indicating a blue color, the reflection of \pm 100 degree was high in the wavelength of $500-600 \mathrm{~nm}$, indicating a green color. For the degrees of \pm 250 , \pm 500 , and \pm 1000 , the reflections of glucose were relatively increased in the red region. The small diagram in Fig. 2 shows the amount of reflection, integrated in the wavelength $500-600 \mathrm{~nm}$ which can effectively discriminate the each degree of glucose test item. The amount of reflection decreases linearly with increasing of the test degree of glucose, and hence the differences of test degrees are quantitatively discriminated.

\subsection{Spectral power distribution of chip-LED}

Figure 3 shows the spectral power distributions of chip LED (E1S10-1W) measured in the wavelength of $400-700 \mathrm{~nm}$. The red-LED starts to emit a red light at 1.7 $\mathrm{V}$, and then arrived at saturated emission at $2.00 \mathrm{~V}$. The

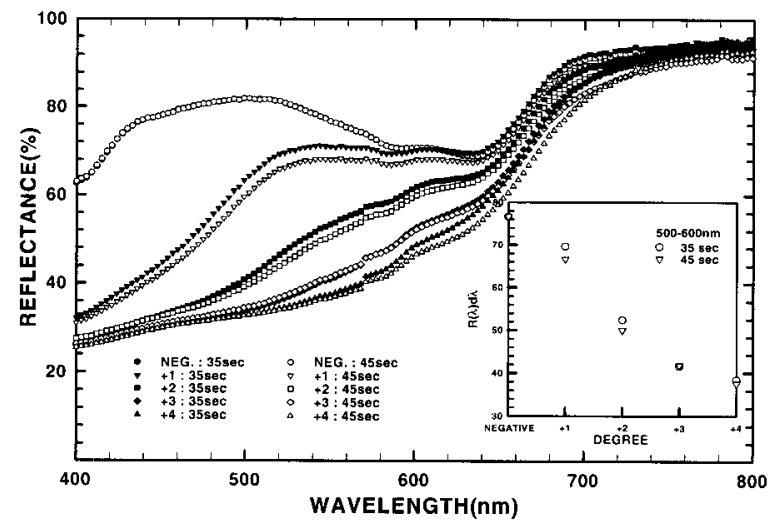

Fig. 2. Reflection spectra of glucose.

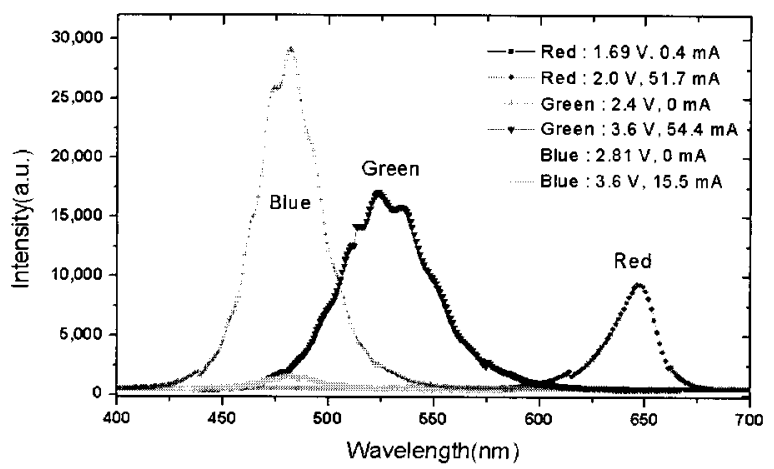

Fig. 3. Spectral power distribution of chip LED E1S10$1 \mathrm{~W}$. peak wavelength of the red LED was $645 \mathrm{~nm}$. The green-LED starts to emit a green light at $2.4 \mathrm{~V}$, and then arrived at saturated emission at $3.6 \mathrm{~V}$. The peak wavelength of the green LED was $520 \mathrm{~nm}$. The blueLED starts to emit a blue light at $2.8 \mathrm{~V}$, and then arrived at saturated emission at $3.6 \mathrm{~V}$. The peak wavelength of the blue-LED was $475 \mathrm{~nm}$. The luminous intensity of blue-LED is larger than those of red-LED and greenLED. Therefore, it is reasonable to correct the difference in luminous intensities of the R, G, and B LED because of the difficulty in color selection by CIE tristimulus values[7-9].

As the temperature goes up, each spectral power distributions of $\mathrm{R}, \mathrm{G}$, and $\mathrm{B}$ decrease. Whereas the redLED exhibits a large reduction of emission in the wavelength of $620-650 \mathrm{~nm}$, the peak wavelengths shift to longer wavelengths remarkably[10].

\subsection{Spectral sensitivity of photodiode}

Figure 4 shows the spectral sensitivity of photodiode MBC-3026C in the wavelength of $300-900 \mathrm{~nm}$. The temperature variation of the spectral sensitivity is relatively lower than that of the spectral power distributions of LED(R, G, B). Photodiode MBC-3026C exhibited the high spectral sensitivity at 360-680 nm.

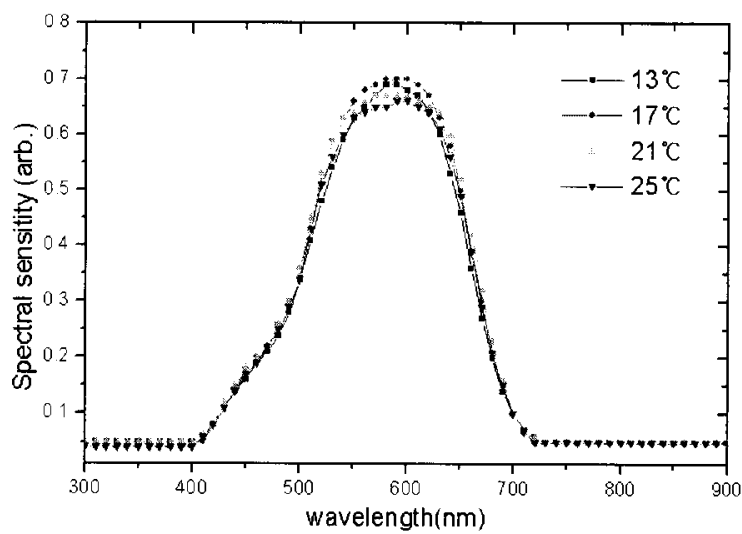

Fig. 4. Spectral sensitivity of photodiode MBC-3026C.

\subsection{Computer simulation \\ 3.4.1 Color stimulus obtained by chip-LED and photodiode}

In a portable-type urine analyzer, the stimulus that the instrument (or brain) interprets as a color is made up of the spectral power distribution of the LEDs, times the spectral reflectance of the urine strip, times the spectral sensitivity of the photodiode. Therefore, color simulation is carried out to discriminate the primary color reaction on the urine strip as follows[11,12]: 


$$
\begin{aligned}
& X=k \int L_{R}(\lambda) R(\lambda) D(\lambda) d \lambda \\
& Y=k \int L_{G}(\lambda) R(\lambda) D(\lambda) d \lambda \\
& Z=k \int L_{B}(\lambda) R(\lambda) D(\lambda) d \lambda
\end{aligned}
$$

Hence $X, Y$, and $Z$ are CIE tristimulus values, $k$ is the normalization factor, $\mathrm{LR}, \mathrm{LG}$ and $\mathrm{LB}$ are the spectral power distributions of $R, G$ and $B$ on the chip-LED (E1S10-1W), $R(\lambda)$ is the reflectance of the urine strip, and $\mathrm{D}(\lambda)$ is the spectral sensitivity of photodiode MBC$3026 \mathrm{C}$.

Using CIE tristimulus values, the CIE chromaticity coordinates describing color stimuli can be obtained as follows:

$$
\begin{aligned}
& x=\frac{X}{X+Y+Z} \\
& y=\frac{Y}{X+Y+Z} \\
& z=\frac{Z}{X+Y+Z}
\end{aligned}
$$

The relation of $x+y+z=1$ holds for all colors. Therefore, the information of $\mathrm{z}$ can be described in $x, y$ chromaticity coordinates.

\subsubsection{The correction of chromaticity coordinates using CMF}

The correction of chromaticity coordinates using CMF is carried out to enhance the accuracy and reproducibility of a portable-type urine analyzer. Standard strip 77 colors were used as color samples in the measurement. CMF of the 1931 CIE standard observer was inserted in the transformation matrix to find a rotational matrix for improving the spectroscopic characteristic of the urine analysis system[13]:

$$
\left[\begin{array}{l}
x \\
y \\
z
\end{array}\right]_{\text {near CIE }}=A *\left[\begin{array}{l}
x \\
y \\
z
\end{array}\right]_{\text {measured value }}
$$

where $\mathrm{A}^{*}$ is the transformation matrix from nonlinear measured values to linear measured values.

The characteristics of the spectral power distribution of the chip-LED and the spectral sensitivity of the photodiode are applied to the color simulation for optimizing the performance of light detecting part in the portable-type urine analyzer. The color matching process is schematically described in Fig. 5. The tristimulus response of the optic detector module composed of the chip-LED and the photodiode can be corrected to match CMF by using the transformation matrix described

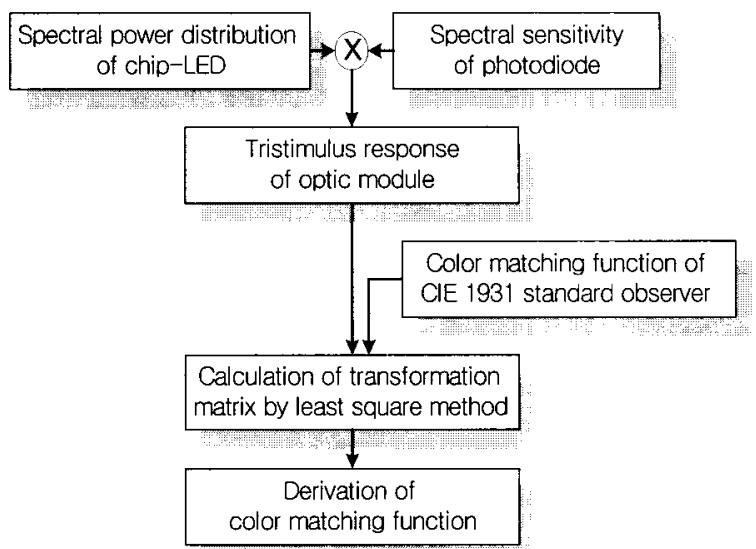

Fig. 5 Transformation of tristimulus values by using CMF.

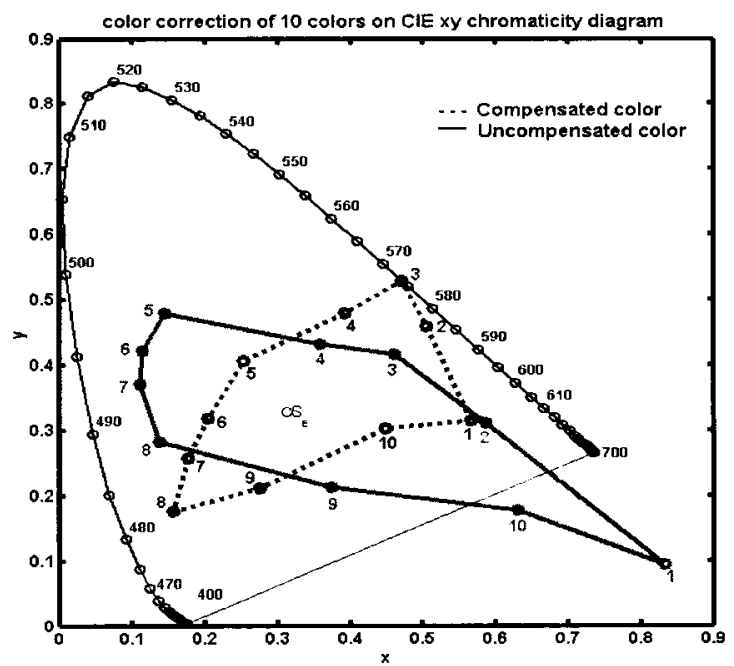

(a)

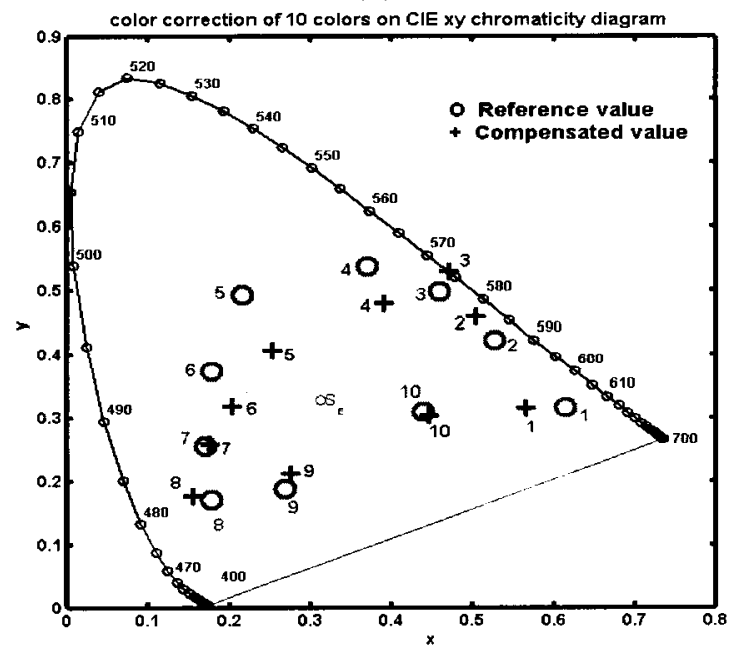

(b)

Fig. 6. (a) Uncorrected and corrected chromaticity coordinates of measured values for standard samples. (b) Reference values by Munsell chromaticity coordinate and corrected values obtained by our urine system. 
Table 2. The mean and standard deviation of measured values for each degree of 9 test items.

\begin{tabular}{|c|c|c|c|c|c|c|c|c|c|c|c|c|c|c|c|}
\hline \multirow{2}{*}{\multicolumn{2}{|c|}{${ }_{\text {ltem }}^{\text {Degree }}$}} & \multicolumn{2}{|l|}{1} & \multicolumn{2}{|l|}{2} & \multicolumn{2}{|l|}{3} & \multicolumn{2}{|l|}{4} & \multicolumn{2}{|l|}{5} & \multicolumn{2}{|l|}{6} & \multicolumn{2}{|l|}{7} \\
\hline & & Mean, std & $\%$ & Mean, std & $\%$ & Mean, std & $\%$ & Mean, std & $\%$ & Mean, std & $\%$ & Mean, std & $\%$ & Mean, std & $\%$ \\
\hline \multirow{3}{*}{$\begin{array}{l}\text { Jrobilino } \\
\text {-gen }\end{array}$} & $R$ & $1971,8.4$ & 1.26 & $1956,18.3$ & 2.75 & $1937,25.6$ & 3.84 & $1901,14.7$ & 2.21 & $1830,46.5$ & 6.98 & $1736,44.5$ & 6.68 & & \\
\hline & G & $1864,6.6$ & 2.99 & $1648,17.5$ & 2.90 & $1486,22.4$ & 3.36 & $1253,20.6$ & 3.09 & $993,58.6$ & 8.79 & 2.9 & 7.94 & & \\
\hline & $B$ & $2082,16.7$ & 2.51 & $1595,24.5$ & 3.05 & $1313,45.0$ & 6.27 & $968,24.1$ & 3.62 & $659,66.4$ & 9.96 & 492 & & & \\
\hline \multirow{3}{*}{ Glucose } & $R$ & $1250,17.6$ & 2.64 & $1179,23.3$ & 3.20 & $1098,12.0$ & 1.80 & $1005,23.8$ & 3.57 & $980 . \quad 5.5$ & 0.83 & & & & \\
\hline & $G$ & $1492,14.0$ & 2.10 & $914,15.2$ & 2.28 & $632,30.2$ & 4.53 & $446,38.9$ & 5.84 & $412,20.7$ & 3.11 & & & & \\
\hline & $B$ & $1800,26.3$ & 3.95 & $510,23.0$ & 3.45 & $259,10.1$ & 1.52 & $163,14.5$ & 2.18 & 148. 4.0 & 0.60 & & & & \\
\hline \multirow{3}{*}{ Ketone } & R & $1859,10.2$ & 1.53 & $1815, \quad 2.5$ & 0.38 & $1672, \quad 5.4$ & 0.81 & $1375,17.2$ & 2.58 & $1123,32.1$ & 4.82 & & & & \\
\hline & G & $1598, \quad 2.6$ & 0.29 & $1232, \quad 7.0$ & 1.05 & $885,12.2$ & 1.83 & $540,13.7$ & 2.06 & 381.22 .1 & 3.32 & & & & \\
\hline & B & $1652,6.8$ & 1.02 & $1315,9.0$ & 1.35 & $969,12.1$ & 1.82 & $595,14.5$ & 2.18 & $412,23.2$ & 3.48 & & & & \\
\hline \multirow{3}{*}{ Bilirubin } & R & 2065.27 .7 & 4.16 & $1892,9.8$ & 1.47 & $1734,17.1$ & 2.57 & $1519,13.0$ & 1.95 & & & & & & \\
\hline & G & $1839,21.2$ & 3.18 & $1573,11.6$ & 1.74 & $1311,19.1$ & 2.87 & $960,29.6$ & 4.4 & & & & & & \\
\hline & $B$ & $2021,27.9$ & 4.19 & $1841,12.1$ & 1.82 & 1646.21 .3 & 3.20 & $1299,36.0$ & 5.40 & & & & & & \\
\hline \multirow{3}{*}{ Protein } & $R$ & $1815,13.8$ & 2.07 & $1515,2.7$ & 0.41 & $1268,43.0$ & 6.45 & $991,2.1$ & 0.32 & $780,13,0$ & 1.95 & $643,18.6$ & 2.79 & & \\
\hline & $G$ & $1687, \quad 5.5$ & 0.83 & 1583.8 .7 & 1.31 & $1464,21.4$ & 3.21 & $1354,2.2$ & 0.33 & $1231,11.9$ & 1.79 & 1139.23 .6 & 3.54 & & \\
\hline & $B$ & $1014,15.2$ & 2.28 & $996,21.3$ & 3.20 & 11.8 & 1.77 & $984, \quad 4.0$ & 0.60 & $995,14.6$ & 2.19 & 7.6 & 2.64 & & \\
\hline \multirow{3}{*}{ Nitrite } & R & $1968,10.5$ & 1.58 & $2006,17.2$ & 2.58 & $1950,22.4$ & 3.36 & & & & & & & & \\
\hline & $G$ & $1868,4.6$ & 0.69 & $1797, \quad 9.4$ & 1.41 & $1641,14.6$ & 2.19 & & & & & & & & \\
\hline & $B$ & $2158,16.8$ & 2.52 & $2112,23.5$ & 3.53 & $2003,14.6$ & 2.18 & & & & & & & & \\
\hline \multirow{3}{*}{$\mathrm{pH}$} & $R$ & $1795,20.9$ & 3.14 & $1429,20.1$ & 3.02 & $1088,6.4$ & 0.96 & $632,15.9$ & 2.39 & 347. & 0.81 & $265,4.4$ & 0.66 & & \\
\hline & $G$ & $999, \quad 8.4$ & 1.26 & $1172,23.2$ & 3.48 & $1157,6.2$ & 0.93 & $1007,17.5$ & 2.63 & $784,9.0$ & 1.35 & $682,10.3$ & 1.55 & & \\
\hline & $B$ & $669,11.8$ & 1.77 & $734,22.2$ & 3.33 & $743,4.1$ & 0.62 & $793,17.8$ & 2.67 & $853,6.3$ & 0.95 & $1021,15.3$ & 2.30 & & \\
\hline \multirow{3}{*}{$\begin{array}{l}\text { Occult } \\
\text { blood }\end{array}$} & $R$ & $1699,24.0$ & 3.60 & $919,35.7$ & 5.36 & $532,23.8$ & 3.57 & 270.23 .6 & 3.54 & & & & & & \\
\hline & G & $1678,14.9$ & 2.24 & $1368,23.0$ & 3.45 & $946,28.0$ & 4.20 & 563.34 .4 & 5.1 & & & & & & \\
\hline & $\mathrm{B}$ & $1465,36.7$ & 5.51 & $1350,21.6$ & 3.24 & $1159,31.0$ & 4.65 & $858,25.9$ & 3.89 & & & & & & \\
\hline \multirow{3}{*}{$\begin{array}{l}\text { Specific } \\
\text { gravity }\end{array}$} & $R$ & $288,20.5$ & 3.08 & $423,13.4$ & 2.01 & $548,12.2$ & 1.83 & $779,9.6$ & 1.44 & 974.21 .6 & 3.24 & $1195,27.9$ & 4.19 & $1430,38.1$ & 5.72 \\
\hline & G & $489,20.4$ & 3.06 & $590,22.8$ & 3.42 & $673,10.8$ & 1.62 & $795, \quad 1.1$ & 0.1 & $857,20.1$ & 3.02 & $929, \quad 9.5$ & 1.43 & & \\
\hline & B & $581,18.5$ & 2.78 & $454,16.4$ & 2.46 & $415, \quad 7.4$ & 1.11 & $406,10.4$ & 1.56 & $383,9.2$ & 1.38 & $376, \quad 2.2$ & 0.33 & $387,11.0$ & \\
\hline
\end{tabular}

above. The differences of the peak wavelengths between CMF and the tristimulus response of the portable-type urine analyzer are due to the relative differences of luminous intensities of $R, G$ and $B$ in the chip-LED.

\subsubsection{Uncorrected and corrected chromaticity coordinates}

Figure 6(a) shows the uncorrected and corrected chromaticity coordinates of the measured values for standard samples. The solid line shows the measured values obtained by the portable-type urine analyzer. The dashed line indicates the corrected values by color simulation using CMF. Figure 6 (b) shows the reference values by Munsell chromaticity coordinates and corrected values obtained by our urine system[11].

Circles denote the chromaticity coordinates of the standard samples by Munsell whereas points denote the chromaticity coordinates of measured values by using the urine analyzer and then corrected by using CMF. The deviations of chromaticity coordinates in Fig. 6 (b) are due to the difference of peak wavelengths between spectral power distributions of chip-LEDs and tristimulus values of CMF.

\subsection{Measured values by the portable-type urine analyzer}

The tristimulus values of the urine strip produced by
Chung-do pharmacy were measured by the developed portable-type urine analyzer. Table 2 shows the mean and standard deviation of the measured values for each degree of 9 test items. Standard deviations increased with increasing the degree of test items. The cause of large deviations is due to low diffusion light occurring from the pads of urine strip. Low diffusion light reduces the sensitivity of the photodiode. The tristimulus values obtained by our urine system exhibited the average accuracy above $95 \%$.

\section{CONCLUSION}

Reflection spectra were measured for each degree of 10 test items in a urine strip to develop the portable-type urine analyzer. The spectral responses composed of the spectral power distribution of the chip-LED and the spectral sensitivity of the photodiode differ significantly from those composed of the standard illuminant and $\mathrm{CMF}$ in human vision. Therefore, computer simulations were carried out to quantitatively discriminate the color reactions of a urine strip in the portable-type urine analyzer. The tristimulus response of the optic detector module composed of the chip-LED and the photodiode could be corrected to match the CMF by using the transformation matrix. The tristimulus values obtained by our urine system exhibited an average accuracy above $95 \%$. 


\section{ACKNOWLEDGEMENTS}

This study was supported by a grant (HMP-98-G-1027) of the HAN (Highly Advanced National) Project, Ministry of Health and Welfare, R. O. K.

\section{REFERENCES}

[1] D. Hernadez, G. Olade, E. Milcent, and A. Beck, Applied Optics, Vol. 34, p. 7952, 1995.

[2] K. R. Jun, S. J. Lee, B. C. Choi, S. H. An, K. Ha, J. Y. Kim, and J. H. Kim, "A study on the development of urine analysis system using strip and evaluation of experimental result by means of fuzzy inference", J. of KOSOMBE(in Korean), Vol. 19, No. 5, p. 477, 1998.

[3] M. J. Vrhel and H. J. Trussell, "Color scanner calibration via a neural network", IEEE ICASSP '99, Vol. 6, p. 3465, 1999.

[4] S. S. Kang, J. H. Kim, H. W. Lee, C. W. Mun, and S. H. Nam, "X-ray response characteristic of $\mathrm{Zn}$ in the polycrystalline Cd1-xZnxTe detector for digital radiography", Trans. on EEM, Vol. 3, No 2, p. 28, 2002.

[5] H. J. Lee, K. T. Lee, G. G. Park, U. S. Park, and H. J. Kim, "Electrical and leakage current characteristic of high temperature polycrystalline silicon thin film transistor", J. of KIEEME(in Korean), Vol. 11, No. 10, p. 918, 1998.

[6] J. H. Kim, J. B. Kim, J. W. Cho, S. C. Kim, B. M. Jin, S. J. Lee, K. W. Kim, and K. R. Jeon, "Computer simulation for spectroscopic analysis of urine strips", Ungyong Mulli (The Korean Physical Society), Vol. 11, No 6, p. 666. 1998.

[7] M. J. Vrhel, "An LED based spectrophotometric instrument", Proc. of SPIE, Vol. 3648, p. 226, 1999.

[8] J. H. Kim, S. B. Lee, G. R. Jeon, S. J. Lee, and J. H. Kim, "Analysis of color reaction on a urine strips", 4th Asia-Pacific Biomedical and Bioengineering Conferences, Seoul, PS-176, p. 456, 1999.

[9] J. H. Kim, J. B. Kim, K. S. Lee, S. J. Lee, and G. R. Jeon, "Color compensation of chromaticity coordinates for urine strips", Sae Mulli (The Korean Physical Society), Vol. 43, No 4, p. 253. 2001.

[10] S. J. Lee, "Implementation of portable urine analysis system using the transformation of chromaticity coordinates and a classification method", Pusan National University, Ph.D thesis(in Korean), p. 76, 2002.

[11] F. W. Billmeyer and Jr. M. Salzman, "Principle of Color Technology", John Wiley \& Sons, Inc., New York, p.1, 1981.

[12] E. J. Giorgianni and T. E. Madden, "Digital Color Management", Addison -Wesley, Massachusetts, p.
3, 1998.

[13] G. Wyszecki and W. S. Stiles, "Color Science", John Wiley \& Sons, Inc., Chapter 8, 1982. 